\title{
Sécurité et traitement des données personnelles
}

Quels impacts sur les réfugiés et les demandeurs d'asile?

Security and processing of personal data: what is the impact on refugees and asylum seekers?

\section{Mathijs Le Rutte}

\section{(2) OpenEdition}

\section{Journals}

\section{Édition électronique}

URL : http://journals.openedition.org/conflits/17793

DOI : $10.4000 /$ conflits. 17793

ISSN : $1777-5345$

\section{Éditeur :}

CCLS - Centre d'études sur les conflits lilberté et sécurité, L'Harmattan

\section{Édition imprimée}

Date de publication : 15 décembre 2009

Pagination : 87-100

ISBN : 978-2-296-11655-9

ISSN : $1157-996 X$

\section{Référence électronique}

Mathijs Le Rutte, "Sécurité et traitement des données personnelles », Cultures \& Conflits [En ligne], 76। hiver 2009, mis en ligne le 03 mai 2011, consulté le 30 mars 2021. URL : http://

journals.openedition.org/conflits/17793; DOI : https://doi.org/10.4000/conflits.17793 


\section{Sécurité et traitement des données personnelles}

Quels impacts sur les réfugiés et les demandeurs d'asile ? 1

\section{Mathijs LE RUTTE}

Mathijs Le Rutte est directeur du Haut Commissariat des Nations unies pour les réfugiés $(H C R)$ au Liban.

Si une personne ou un groupe de personnes est persécuté, il lui est possible de fuir vers un lieu plus sûr. En revanche, si la population mondiale dans son ensemble est menacée, où peut-elle aller ? La menace du terrorisme a modifié les attitudes, les perceptions et les mécanismes d'adaptation. Opposant la sécurité à la liberté, elle a créé une polarisation entre ceux qui prétendent que la recherche de la sécurité justifie la limitation des droits et des libertés et ceux qui pensent que rien ne justifie l'atteinte aux libertés fondamentales ${ }^{2}$.

Il est fréquent aujourd'hui que les gouvernements et leurs agences de renseignement aient recours à un éventail de techniques d'enquête spéciales, telle que la collecte d'informations à grande échelle, qui concernent particulièrement les personnes soupçonnées d'activités criminelles comme le terrorisme. Ces techniques sont justifiées par l'objectif et l'obligation qu'ont les Etats de protéger leurs habitants. Cependant, reste le danger que l'appréciation de ce qui est suspect soit élargie, par inadvertance ou délibérément, en particulier lorsqu'il s'agit de prévenir la criminalité et de détecter l'organisation d'actes criminels.

La nature transnationale du crime organisé et du terrorisme nécessite une collaboration et une coordination étroites entre les agences de renseignement. Cela signifie également que la plupart des données recueillies nécessitent

1. Les opinions et observations exprimées dans cet article sont celles de l'auteur et ne représentent pas nécessairement les points de vue des Nations unies ou du HCR. Mathijs Le Rutte souhaite remercier Tharinda Puth, stagiaire auprès du HCR, pour son aide précieuse apportée dans la recherche, la relecture et l'édition de cet article.

2. "Ceux qui peuvent renoncer à la liberté essentielle pour obtenir un peu de sécurité temporaire, ne méritent ni la liberté ni la sécurité », Benjamin Franklin, 1775. 
d'être largement partagées ; il sera donc difficile de s'assurer que leur utilisation ultérieure réponde au seul but ayant initialement présidé à leur collecte.

L'identité personnelle et la capacité de rassembler tous les faits utiles pour l'identification des personnes constituent un critère fondamental pour le maintien de l'ordre et la lutte contre la criminalité. L'identification est aussi indispensable dans le cadre des contrôles des voyages internationaux.

En plus des répercussions importantes qu'elles entraînent sur la population en général, ces entreprises d'identification touchent en particulier les demandeurs d'asile et les réfugiés. En effet, ces personnes ont besoin de la protection d'Etats autres que celui dont ils sont ressortissants, et leur sécurité dépend de leur capacité à circuler et à voyager. La vulnérabilité des demandeurs d'asile et des réfugiés est d'autant plus marquée qu'ils dépendent de procédures imposées et qu'ils ont généralement un accès limité au contrôle et aux recours judiciaires. Cet article entend surtout attirer l'attention sur la manière dont ces évolutions risquent d'entraîner des effets négatifs sur les demandeurs d'asile et les réfugiés.

\section{L'accès aux procédures d'asile}

Dès lors qu'un réfugié s'enfuie, la relation entre l'Etat et son citoyen se modifie. La fuite est généralement déclenchée par une situation dans laquelle l'Etat ne veut ou ne peut plus protéger les ressortissants concernés. Dans de telles circonstances, le régime international de protection des réfugiés, bien établi et largement reconnu, s'applique. Cela signifie qu'un réfugié dépend désormais de son pays d'asile pour la protection de ses droits civils et de sa dignité humaine. Les divers instruments juridiques relatifs aux droits de l'homme obligent les Etats à respecter et à garantir les droits de toute personne qui se retrouve sur leur territoire ou de toute personne soumise à leur juridiction. Cela signifie que, même si la Convention relative au statut de réfugié de 1951 accorde des protections spécifiques aux réfugiés, tout Etat, partie à la Convention ou non, a des obligations vis-à-vis des réfugiés ${ }^{3}$.

Le processus de demande d'asile constitue généralement la première étape officielle de cette nouvelle relation. Une personne est qualifiée de « réfugiée »

3. Voir Comité des droits de l'homme, observations générales, $\mathrm{n}^{\circ} 15$ et n $\mathrm{n}^{\circ} 31$ :

http://www.unhchr.ch/tbs/doc.nsf/(Symbol)/bc561aa81bc5d86ec12563ed004aaa1b ;

http://www.unhchr.ch/tbs/doc.nsf/(Symbol)/CCPR.C.21.Rev.1.Add.13.En? Opendocument

Voir aussi «La Déclaration sur les droits de l'homme des personnes qui ne possèdent pas la nationalité du pays dans lequel elles vivent " adoptée par l'Assemblée générale, résolution 40/144 du 13 décembre 1985. Son article 5 confirme expressément que l'étranger jouit, conformément au droit interne et sous réserve de l'obligation internationale de l'Etat dans lequel il est présent, $\mathrm{du}$ "droit à la protection contre les ingérences arbitraires on illégales dans sa vie privée, sa famille, son domicile ou correspondance ",

http://www.unhchr.ch/html/menu3/b/o_nonnat.htm 
à condition que sa crainte d'une persécution dans son pays d'origine soit fondée. La procédure d'asile n'établit pas le bien-fondé de la crainte, elle ne peut que la confirmer. Les procédures d'asile exigent que le demandeur fournisse les preuves venant démontrer cette crainte. Ainsi, les réfugiés doivent fournir des informations détaillées et personnelles sur leur famille et sur leurs expériences, qui permettront de déterminer le statut de réfugié. Ces informations doivent par ailleurs servir aux associations humanitaires qui ont pour mission de leur offrir soins et assistance.

Cela explique la nécessité d'établir un équilibre entre, d'un côté, la quantité d'informations qui doit être recueillie et partagée pour aider les réfugiés, et de l'autre, les limites qu'il convient de fixer au traitement de ces données afin d'éviter tout accès non-autorisé. La nécessité de protéger les données individuelles et personnelles des réfugiés peut donc être justifiée de deux façons distinctes. Premièrement, les Etats doivent respecter le droit des réfugiés à la vie privée, comme c'est le cas pour toute personne dont les informations sont collectées. Il est important de souligner que les réfugiés, qui ont quitté leur environnement, sont soumis à des processus et à des règlements qu'ils ne comprennent pas nécessairement et qui ne leur indiquent pas toujours clairement leurs droits. Cela risque d'entraver le droit à un accès à la justice qui soit semblable à celui des citoyens du pays d'accueil dans les cas où les réfugiés souhaiteraient engager un recours contre d'éventuelles violations de leur droit à la vie privée. Deuxièmement, la personne qui déclare craindre une persécution dans son Etat d'origine doit bénéficier d'une aide internationale afin d'être protégée contre tout acte que sont susceptibles de perpétrer d'éventuels d'agents persécuteurs.

En aucun cas des informations relatives aux demandeurs d'asile (leurs données personnelles, leur localisation, le fait d'avoir demandé l'asile, d'autres demandes d'asile éventuellement déposées dans d'autres pays, etc.) ne doivent tomber entre les mains de l'Etat persécuteur. Il s'agit là d'un des principes fondamentaux de la protection des réfugiés et des demandeurs d'asile qui s'applique à toutes les étapes de la procédure d'asile. Les conséquences possibles $\mathrm{du}$ non-respect de ce principe sont nombreuses. En effet, un manque de confiance chez le demandeur d'asile peut induire de sa part certains comportements réticents lorsqu'il devra exposer sa situation personnelle ou demander le statut de réfugié. Il y a également le risque que des membres de la famille ou des connaissances du demandeur d'asile restés dans son pays d'origine soient mis en danger; le demandeur d'asile peut être en danger dans le pays d'asile ; le demandeur d'asile peut risquer des représailles lors de son retour éventuel dans son pays d'origine ; et le demandeur d'asile peut devenir un réfugié « sur place ${ }^{4}$ ».

4. Un réfugié «sur place » est un réfugié qui est hors de son pays d'origine, qu'il a quitté pour d'autres raisons que la persécution, mais qui ne peut pas y rentrer en raison d'une crainte fondée de persécution. 
La directive de 2005 du Conseil européen sur la procédure d'asile confirme que les autorités d'asile ne peuvent ni divulguer des informations sur le demandeur d'asile aux agents de persécution, ni rechercher des informations auprès de ces mêmes acteurs ${ }^{5}$.

Reste que les gouvernements manifestent une préoccupation croissante en matière de sécurité nationale. Les efforts visant à lutter contre le terrorisme international ont notamment entraîné la mise en œuvre de contrôles sans précédent portant sur le déplacement et la circulation des personnes. Bien que les enjeux de sécurité soient justifiables, il existe un risque réel que les Etats se servent de ceux-ci pour instituer des mesures qui dérogent aux principes fondamentaux des droits de l'homme. Ce sont surtout les réfugiés qui risquent d'être affectés.

S’il existe un principe juridique général selon lequel la charge de la preuve incombe à la partie requérante, il est largement admis comme pratique équitable que le devoir de vérifier et d'évaluer tous les faits pertinents d'une demande d'asile doit être partagé entre le demandeur et l'examinateur. Les réfugiés, devant généralement fuir leur domicile de façon impromptue, sans préparation préalable et en situation de grande détresse, ne disposent généralement pas de documents personnels ou d'autres pièces justifiant leurs déclarations. Comme il est mentionné dans le Guide HCR des procédures et critères à appliquer pour déterminer le statut de réfugié:

"Aussi, bien que la charge de la preuve incombe en principe au demandeur, la tâche d'établir et d'évaluer tous les faits pertinents sera-t-elle menée conjointement par le demandeur et l'examinateur. Dans certains cas, il appartiendra même à l'examinateur d'utiliser tous les moyens dont il dispose pour réunir les preuves nécessaires à l'appui de la demande 6 ».

5. Directive 2005/85/CE du Conseil du 1er décembre 2005 relative aux normes minimales concernant la procédure d'octroi et de retrait du statut de réfugié dans les Etats membres, article 22, Collecte d'informations relatives à des cas particuliers : "Dans le cadre de l'examen de cas particuliers, les Etats membres : a) ne divulguent pas directement à l'auteur (ou aux auteurs) présumé(s) de persécutions à l'encontre $d u$ demandeur d'asile les informations concernant une demande d'asile, ou le fait qu'une demande d'asile a été introduite; b) ne cherchent pas à obtenir du ou des auteurs présumés de persécutions à l'encontre du demandeur d'asile des informations d'une manière telle que cet ou ces auteurs soi(en)t directement informé(s) qu'une demande d'asile a été introduite par le demandeur en question, et que l'intégrité physique de ce dernier et des personnes à sa charge, ou la liberté et la sécurité des membres de sa famille qui séjournent encore dans son pays d'origine, soient compromises».

http://eur-lex.europa.eu/LexUriServ/LexUriServ.do?uri=OJ:L:2005:326:0013:01:FR:HTML

6. Guide HCR des procédures et critères à appliquer pour déterminer le statut de réfugié au regard de la Convention de 1951 et du Protocole de 1967 relatifs au statut des réfugiés, paragraphe 196. UNHCR, 1979 (réédité, Genève, janvier 1992). Disponible sur :

http://www.unhcr.org/cgi-bin/texis/vtx/refworld/rwmain/opendocpdf.pdf? reldoc=y\&amp;docid $=471332 \mathrm{~d} 22$ 
Pareillement, la directive du Conseil européen relative aux normes minimales pour la qualification au statut de réfugié prescrit qu'il «appartient à l'Etat membre d'évaluer, en coopération avec le demandeur, les éléments pertinents de la demande 7 ».

Une vérification des registres d'état civil dans le pays d'origine est évidemment impossible. L'article 25 de la Convention de 1951 relative au statut de réfugié établit l'obligation qui incombe au pays d'asile de fournir une assistance administrative au réfugié, ce qui inclut l'émission de documents et de certificats. La rupture des liens avec le pays d'origine signifie donc que le réfugié ne peut plus compter sur aucune aide de sa part. De plus, il serait inconcevable que le réfugié demande de l'aide, car cela impliquerait un contact avec ses persécuteurs. La crainte fondée du réfugié oblige donc le pays d'asile à le tenir éloigné de tout contact avec des persécuteurs ${ }^{8}$.

Alors que le nombre de réfugiés dans le monde ne cesse d'augmenter, celui des demandeurs d'asile dans l'Union européenne (UE) est en baisse. Le renforcement des contrôles aux frontières devient une dimension importante de la gestion des réfugiés et des demandeurs d'asile en Europe. En outre, parmi ceux qui atteignent l'Europe, un nombre croissant de personnes choisit de rester de manière irrégulière sur le territoire plutôt que d'entamer une procédure d'asile.

Un des aspects inquiétants de cette tendance est le fait que nombre de réfugiés sont contraints de recourir à des moyens irréguliers de voyage comme le recours à des passeurs illégaux ou des trafiquants, ce qui n'est pas sans mettre leur vie en danger pour atteindre l'Europe.

L'UE a développé une gamme de mesures ayant toutes pour objectif de contrôler les flux migratoires au-delà de ses frontières. Ainsi, créée en 2005, l'Agence européenne pour la gestion de la coopération opérationnelle aux frontières extérieures (FRONTEX), est en charge de l'exécution d'un certain nombre de ces mesures. Elles incluent le placement dans les aéroports étrangers d'officiers de liaison des services d'immigration ${ }^{9}$, et des missions spécifiques confiées aux gardes-frontières terrestres telles que l'obligation d'obtenir des visas, l'imposition de sanctions aux transporteurs, la mobilisation de la biométrie et de bases de données dans le domaine de la migration.

7. Chapitre II, article 4, directive 2004/83/CE du Conseil du 29 avril 2004 concernant les normes minimales relatives aux conditions que doivent remplir les ressortissants des pays tiers ou les apatrides pour pouvoir prétendre au statut de réfugié ou les personnes qui, pour d'autres raisons, ont besoin d'une protection internationale, et relatives au contenu de ces statuts,

http://eur-lex.europa.eu/LexUriServ/LexUriServ.do?uri=CELEX:32004L0083:FR:HTML

8. Voir aussi le commentaire sur la Convention relative au statut des réfugiés de 1951, Genève 1963, Atle Grahl-Madsen, article 25.

9. Permettant aux pays de destination d'exercer un contrôle d'accès même avant le départ. 
Les pays devraient envisager de suspendre les restrictions de visas pour les personnes provenant d'un pays dans lequel il y a de graves violations des droits humains, un conflit armé ou d'autres crises humanitaires. Les restrictions de visa apparaissent également excessives pour les personnes en provenance de pays où il n'existe pas de mécanismes appropriés pour la délivrance de tels documents de voyage.

L'introduction de sanctions à l'encontre des opérateurs aériens transportant des personnes sans papiers a déplacé la responsabilité qu'avaient les fonctionnaires d'Etat vers les compagnies aériennes. Alors que les premiers ont été formés pour identifier et mettre en œuvre des demandes d'asile, les transporteurs aériens sont mus par une autre logique : celle d'éviter à tout prix les amendes et les frais d'accompagnement.

$\mathrm{Au}$ cours de ces dernières années, les Etats membres de l'Union européenne ont également eu de plus en plus recours à la pratique de l'affectation dans d'autres pays de personnels en charge des questions d'immigration. Ceci est particulièrement le cas dans les pays d'origine ou de transit, dans lesquels les Etats membres souhaitent assurer un contrôle plus efficace des mouvements migratoires à destination de leur territoire.

En outre, il est souvent très difficile pour les demandeurs d'asile de respecter les critères établis les concernant. En effet, certaines mesures exigent un niveau de preuve bien supérieur à celui que l'on peut raisonnablement attendre des réfugiés, compte tenu des conditions souvent précaires dans lesquelles leur fuite s'est déroulée. De nombreux Etats sanctionnent sévèrement le demandeur d'asile qui est dans l'impossibilité de présenter une pièce d'identité et il peut même, pour cette raison, se voir refuser l'accès à la procédure de demande d'asile. La charge de la preuve peut aussi être augmentée, ce qui a pour conséquence de mettre en cause la crédibilité du demandeur sur la véracité de sa situation, étant donné qu'il ne dispose pas de pièce d'identité. D'autres Etats ont recours à la détention des demandeurs qui sont interpellés sans papiers, ou procèdent même à leur expulsion. Plusieurs pays ont déjà introduit, comme une alternative à la détention et pour faciliter leur renvoi, le marquage électronique des demandeurs d'asile dont la demande a été refusée ${ }^{10}$. Dans d'autres pays, certains hommes politiques ont proposé que tous les demandeurs d'asile soient marqués afin de contrôler leurs déplacements et de prévenir le crime ${ }^{11}$. Pourtant, le fait de ne pas avoir de papiers ne devrait pas constituer un crime. La Convention relative au statut de réfugié de 1951 a pris en compte les difficultés qu'ont les réfugiés à produire les documents

10. Sur le marquage par bracelets électroniques, voir :

http://www.guardian.co.uk/politics/2006/mar/14/immigrationandpublicservices; et : http://www.abc.net.au/worldtoday/stories/s334626.htm

11. Voir, Rydgren J. (ed.), Movements of Exclusion: Radical Right-Wing Populism in the western World, New York, Nova Science Publishers, 2005. 
requis, en interdisant aux Etats de sanctionner pénalement leur entrée ou leur séjour irréguliers ${ }^{12}$.

Compte tenu de cette incapacité des réfugiés de parvenir à un niveau de preuve plus élevé, un autre principe de procédure existe depuis longtemps pour pallier ce manque. Dans les cas où les déclarations du demandeur d'asile sont, dans l'ensemble, cohérentes et plausibles, le bénéfice du doute lui est accordé même s'il ne peut fournir certains éléments de preuve. Cependant, il semble qu'actuellement le contexte du «tout-sécuritaire » ait favorisé le doute, en d'autres termes la suspicion, au préjudice du demandeur d'asile.

La tendance à criminaliser l'asile constitue aussi un phénomène inquiétant et éventuellement dangereux. Les pays d'asile potentiels craignent de plus en plus que les réfugiés soient des agents représentant leur gouvernement, plutôt que des personnes cherchant véritablement une protection contre des régimes répressifs qu'ils fuient. Dans le contexte européen par exemple, il existe un certain nombre de systèmes d'informations dans les domaines de l'asile, de la migration et du maintien de l'ordre et de la justice ${ }^{13}$. Les propositions en vue de leur interopérabilité et le partage des données entre les Etats membres et avec des Etats tiers, compromettent gravement les principes de protection des données et peuvent également nuire à la sécurité des réfugiés. Ainsi, en octobre 2007, la Commission européenne a invité le HCR à un débat sur une proposition législative visant à permettre à la police et d'autres services de maintien de l'ordre d'accéder à la base de données EURODAC ${ }^{14}$. Cet accès a alors été proposé «à des fins de consultation dans le cours de l'exercice de leurs fonctions dans les domaines de la prévention, de la détection et de l'investigation des crimes terroristes et autres crimes graves ".

12. Voir la Convention relative au statut de réfugié de 1951, article 31(1) Réfugiés en situation irrégulière dans le pays d'accueil : "Les Etats contractants n'appliqueront pas de sanctions pénales, $d u$ fait de leur entrée ou de leur séjour irréguliers, aux réfugiés qui, arrivant directement du territoire où leur vie ou leur liberté était menacée au sens prévu par l'article premier, entrent ou se trouvent sur leur territoire sans autorisation, sous la réserve qu'ils se présentent sans délai aux autorités et leur exposent des raisons reconnues valables de leur entrée ou présence irrégulières ".

13. Le système d'information Schengen de deuxième génération (SIS II), le système d'information sur les visas (VIS), le système d'information d'asile européen (EURODAC).Voir : http://europa.eu/legislation_summaries/justice_freedom_security/police_customs_cooperation/133502_fr.htm

14. Institué en décembre 2000, EURODAC constitue une base de données grâce à laquelle sont collectées, à des fins de comparaison, les empreintes digitales de tout étranger de plus de 14 ans déposant une demande d'asile et celles des immigrants illégaux. Sur EURODAC, voir notamment Irma Van der Ploeg, "Eurodac” and the Illegal Body. The Politics of Biometric Identity ", Ethics and Information Technology, vol. 1, n 4, 1999, pp. 37-44 ; Evelien Brouwer, "Eurodac : its Limitations and Temptations ", European Journal of Migration and Law, vol. 4, $\mathrm{n}^{\circ}$ 2, 2002, pp. 231-247 ; Jonathan P. Auss, "Supranational Governance in an "Area of Freedom, Security and Justice": Eurodac and the Politics of Biometric Control ", University of Sussex, Sussex European Institute, SEI working paper $\mathrm{n}^{\circ} 72,2003$ : http://www.sussex.ac.uk/sei/documents/wp72.pdf 
Même en dehors du fait que les informations disponibles dans la base de données semblent être mal adaptées aux besoins que les services de l'ordre avaient exprimés, cette utilisation irait, pour sûr, bien au-delà de l'objectif initialement dévolu à cette base de données d'empreintes digitales. Lequel était à l'origine strictement limité à la détermination de l'Etat responsable d'une demande d'asile déposée dans un Etat membre de l'UE. L'exploitation de ces informations pour d'autres raisons constitue, en matière de protection des données, un détournement du principe de finalité ${ }^{15}$. De surcroît, l'utilisation des données des demandeurs d'asile dans le cadre de la lutte contre le terrorisme créerait une stigmatisation et une discrimination de ce groupe. En 2006, la Cour constitutionnelle d'Allemagne a d'ailleurs reconnu que la pratique de profilage des données par rapport à des critères établis par la police allemande constituait une violation de la Constitution. Elle a souligné que cette pratique risquait de soumettre les groupes visés à des mesures de contrôle additionnelles, et de confirmer les préjugés de la société envers les groupes affectés ${ }^{16}$.

Dans la vente de leurs produits, les entreprises qui développent des systèmes de sécurité informatisés n’hésitent pas à exploiter ces craintes. Le site Internet de l'une de ces sociétés annonce : "La hausse de la criminalité; Schengen; EURODAC - le monde change et avec lui les exigences de ses forces

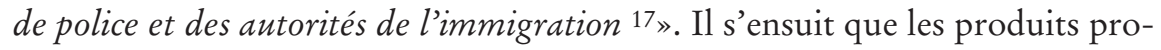
posés par ces entreprises deviennent pour les autorités des outils considérés comme essentiels afin de «lutter» contre ces nouveaux défis.

De plus, le partage et l'échange d'informations par l'intermédiaire d'Interpol présente une opportunité pour les Etats persécuteurs d'abuser de ce mécanisme de poursuite. En délivrant des mandats d'arrêt mal fondés ou en fournissant d'autres informations incriminantes, ces données peuvent entrâner des persécutions même au-delà de la frontière. Le résultat de ces échanges d'information peut ainsi priver les ressortissants de la protection internationale dont ils auraient besoin.

Ces développements montrent bien qu'il existe une volonté de la part des décideurs politiques d'établir un lien entre l'asile et la criminalité. Bien qu'il y ait sans doute des éléments criminels qui cherchent à abuser le système d'asile, le fait de présupposer que les demandeurs d'asile sont suspects par définition est à la fois totalement erroné et dangereux. En outre, l'établissement d'un tel lien aura pour effet de contraindre de plus en plus les demandeurs d'asile à utiliser des canaux illicites d'entrée et de séjour dans les pays d'asile.

15. Sur ce point, et en particulier les réactions qu'il suscite de la part du Contrôleur européen de la protection des données, voir Piazza P., "L’Europe biométrique contre les libertés ", Regards sur l'actualité, $\mathrm{n}^{\circ}$ 349, mars 2009, p. 67-68.

16. Jugement de la Cour constitutionnelle, 4 avril 2006, 1 BvR 518/02. : http://www.bundesverfassungsgericht.de/entscheidungen/rs20060404_1bvr051802.html

17. http://www.steria.no/fit/index.db2 ?id $=45$ 
Il existe un risque réel que les phénomènes discutés ci-dessus, c'est-à-dire les mesures de contrôle pour entrer sur le territoire, les restrictions dans les procédures d'asile et la tendance à criminaliser l'asile, remettent en cause le droit fondamental de demander l'asile. Plus grave encore, ils entraînent un sérieux risque de violation du principe fondamental de non-refoulement.

\section{Protection des données des réfugiés}

Après qu'une personne s'est vue accorder le statut de réfugié, le traitement de ses données à caractère personnel, utilisées à des fins de vérification d'identité et de contrôle, risque de porter atteinte à la libre jouissance des droits des réfugiés. En 2004, le HCR a créé le Profile Global Registration System (proGres), un système d'enregistrement des réfugiés globalement standardisé. Ce système est opérationnel dans soixante-trois pays et détient à tout moment plus de 4,5 millions de dossiers individuels. Le HCR recueille des informations dans le but de mieux adapter les demandes de protection en fonction des besoins de chacun et de trouver des solutions les plus durables et appropriées pour chacun. Le mandat de protection spécifique du HCR nécessite la collecte et le traitement de données pour une variété de services et de processus essentiels. Ces derniers incluent la délivrance de pièces d'identité, la détermination du statut de réfugié, la planification et le ciblage de l'assistance humanitaire et des services, la délivrance de documents permettant l'accès à des services (cartes de rationnement, cartes de santé), l'identification des bénéficiaires ayant des besoins spéciaux, la recherche de solutions durables telles que le rapatriement volontaire, la réinstallation et l'intégration locale.

Pour des raisons de cohérence, de coordination et d'opérationnalité, il est important de considérer la possibilité de rendre ces bases de données accessibles par Internet. Cela n'est toutefois pas sans entraîner des risques d'atteinte à la protection des données à caractère personnel. En effet, avec cette pratique, le risque d'interférences ou de pertes, accidentelles ou malveillantes, augmente. Par conséquent, tout doit être soigneusement réglementé et à tout moment respecté pour assurer les obligations de protection des données vis-àvis des réfugiés.

Il va sans dire que les Etats, particulièrement les pays d'asile et les pays de réinstallation, ont un intérêt justifié à avoir accès à certaines informations sur les réfugiés afin de les protéger. Etant donné que les méthodes de collecte de données sont de plus en plus avancées et, par conséquent, les informations accumulées par le HCR de plus en plus précises, d'autres Etats ont un intérêt croissant à avoir accès à ces informations. Les administrations justifient cet intérêt par leurs préoccupations en matière de lutte contre le terrorisme, le crime organisé et la fraude, de contrôle des mouvements irréguliers, et d'identification des personnes ne pouvant pas bénéficier de la protection conférée aux réfugiés. 
Il est clair que tout partage de données doit être effectué dans le respect des principes régissant la protection des données. De plus, sans le consentement préalable de la personne concernée, le HCR n'est pas habilité à partager l'information pour des fins autres que celles pour lesquelles elles ont été recueillies.

En outre, il existe une tension entre d'un côté, les normes d'identification d'enregistrement du HCR, fondées sur les difficultés qu'ont les réfugiés à fournir des papiers d'identité et d'autres éléments de preuve, et de l'autre côté, la nécessité de satisfaire des conditions de plus en plus strictes qu'imposent les gouvernements en fonction de leurs préoccupations en matière de sécurité.

L'utilisation de la technologie biométrique est particulièrement encouragée par certains gouvernements afin de compenser un certain manque de précision dans l'identification d'une personne par les moyens conventionnels ${ }^{18}$. Par ailleurs, le HCR a introduit un projet pilote consistant à utiliser des empreintes digitales dans huit de ses opérations avec, pour but principal, la réduction du double enregistrement. Il évalue actuellement l'utilité et l'efficacité du recours à cet identifiant. Bien qu'il soit nécessaire d'entreprendre de plus amples recherches en la matière, nous constatons que la précision et la fiabilité du système dactyloscopique ne sont pas aussi incontestables que nous aurions pu le croire.

Début 2008, les Etats-Unis ont introduit les tests ADN pour des Somaliens soumis à une réinstallation ${ }^{19}$ par le HCR en réponse à des soupçons de fraude. Le programme de réinstallation a été suspendu quand de nombreuses familles ont obtenu des résultats négatifs, malgré leurs déclarations selon lesquelles des liens de sang existaient entre leurs membres. Dans de tels cas, le HCR est préoccupé par les conséquences d'une mauvaise appréciation d'un contexte culturel spécifique. Vu le contexte particulier des filiations familiales au sein de la communauté somalienne, il est difficile d'imposer une preuve aussi rigoureuse que celle des liens de sang. Les Somaliens intègrent fréquemment dans leur famille les orphelins, les mineurs non-accompagnés, ou les enfants nés hors mariage ou après un viol. Ceux-ci constituent une véritable famille, et les raisons justifiant la volonté de non divulgation du véritable lien de parenté sont souvent légitimes. Le HCR a récemment pris position sur l'utilisation de l'ADN dans les situations de regroupement familial des réfugiés de façon à mettre en évidence ses implications en matière de droits de l'homme, en particulier en ce qui concerne le droit à la vie privée 20.

18. Voir notamment Crettiez X. et Piazza P. (dir.), Du papier à la biométrie. Identifier les individus, Paris, Presses de Sciences Po, 2006.

19. La réinstallation est l'une des trois solutions durables au problème des réfugiés, permettant à ceux qui correspondent à des critères spécifiques basés sur leur vulnérabilité, de s'installer dans un pays tiers.

20. UNHCR, "Note on DNA Testing to Establish Family Relationships in the Refugee Context », juin 2008 : http://www.unhcr.org/refworld/docid/48620c2d2.html 
La Convention relative au statut de réfugié de 1951 contient des dispositions qui régissent le voyage international des réfugiés. Les pays d'asile émettent, à cette fin, des titres de voyage aux réfugiés. Le HCR émet également de tels documents au nom des pays d'asile qui n'ont pas la capacité de le faire. Ces documents sont dénommés « Documents de Voyage de la Convention » (ou DVC). Un modèle quelque peu anachronique de ce document est inclus dans l'annexe de cette convention. L'annexe 9 de la Convention de Chicago sur l'aviation civile internationale prévoit que tous les Etats contractants devraient être en mesure de délivrer des passeports lisibles par une machine appropriée dès le $1^{\text {er }}$ avril 2010. Le HCR témoigne d'un vif intérêt pour ce projet de l'Organisation de l'aviation civile internationale destiné à aider les Etats à se préparer à émettre de nouveaux passeports. Il est important de trouver les moyens d'inclure dans ce projet des mesures concernant l'assistance à l'émission des DVC pour les réfugiés. Il sera aussi pertinent d'étudier la façon dont le standard des DVC, défini dans la Convention relative au statut de réfugié de 1951, ainsi que dans la Convention sur le statut des apatrides de 1954 qui a des dispositions similaires, peut être mis en conformité avec les normes de la Convention de Chicago relative à l'aviation civile internationale.

\section{Que peut faire le HCR ?}

Tel que mentionné précédemment, le mandat du HCR, qui consiste à protéger les réfugiés, nous confronte à un défi dans le domaine de la protection des données. La protection des réfugiés nécessite d'une part la collecte et le partage des données individuelles, et d'autre part, la protection de leurs données contre tout accès non-autorisé, particulièrement de ceux qui sont les plus susceptibles de leur causer un préjudice. Les principes directeurs des Nations unies concernant les fichiers informatisés de données à caractère personnel, adoptés par l'Assemblée générale en 1990, fournissent un cadre pour la protection des données au sein d'une organisation comme le HCR, ainsi que pour le partage de données avec des acteurs tiers. Le HCR a adapté ces principes dans un cadre qui lui est propre. Néanmoins, le développement des technologies informatiques et les pressions, déjà évoquées, qu'exercent certains gouvernements au nom de préoccupations sécuritaires, imposent un renforcement et une mise à jour régulière de ce cadre. Le HCR a aussi créé un Groupe de travail de gestion de données rassemblant des spécialistes dans les domaines opérationnels, juridiques et des technologies de l'information pour améliorer ce cadre.

Le mandat particulier du HCR nous oblige donc à respecter les principes de protection de données de façon très stricte. La protection de la personne virtuelle doit être réalisée de façon aussi précise et rigoureuse que celle de la personne physique. Les principes directeurs des Nations unies incluent une clause humanitaire indiquant qu'une dérogation à ces principes peut être expressément prévue lorsque l'objet du fichier est la protection des droits de 
l'homme et des libertés fondamentales de la personne concernée, ou l'aide humanitaire. Cette clause nous est donc nécessaire afin de pouvoir qualifier les dérogations à certaines obligations, telle que l'autorisation pour la collecte des données raciales ou d'origine ethnique, la vie sexuelle, les opinions politiques et religieuses, et pour limiter l'accès aux personnes concernées. Il est d'autant plus important que les conditions dans lesquelles le HCR utilise ces dérogations soient bien précisées et respectées.

Le HCR dispose d'un statut d'organisation internationale et il est ainsi soumis à la réglementation portant sur les privilèges et les immunités diplomatiques. Ceci protège les documents et les données du HCR contre l'ingérence des gouvernements. Des règlements nationaux additionnels sont souvent nécessaires pour régir le traitement de données entrepris par les partenaires de gestion. Bien que l'immunité diplomatique du HCR puisse, en principe, être étendue à des données qui sont administrées par ces organismes, ni ces organismes, ni leurs fonctionnaires ne peuvent jouir de privilèges et autres immunités. Cela laisse donc ces partenaires exposés à des interférences et des poursuites.

En ce qui concerne les responsabilités du personnel du HCR vis-à-vis des réfugiés, les règlements internes visent à garantir le strict respect des principes de protection des données en faveur des réfugiés, et engagent la responsabilité du personnel en cas de méconnaissance de ces principes ${ }^{21}$.

Les principes directeurs des Nations unies recommandent que chaque organisme désigne un superviseur ou un contrôleur dans le domaine de la protection des données. Malheureusement, le HCR ne dispose pas d'un tel poste : il est difficile d'en proposer la création étant donné le défi budgétaire auquel fait face l'organisation. Cependant, il serait fortement souhaitable que le HCR impose ce niveau de contrôle et de responsabilité, la protection des données étant au cœur de son mandat de protection des réfugiés.

Au-delà de la protection des données des réfugiés au sein du HCR et du partage de ces données avec les tiers, il existe une importante dimension externe du mandat du HCR à l'égard de la protection des données. Les tendances et les évolutions discutées précédemment ont un impact direct ou indirect sur les droits des demandeurs d'asile et des réfugiés. Tel que nous avons tenté de l'expliquer dans cet article, des exigences trop strictes en matière

21. De plus, le Statut du personnel de l'Organisation des Nations unies, article 1(2) stipule que "(i) Les membres du personnel doivent observer la plus grande discrétion sur toutes les questions officielles. Sauf, le cas échéant, dans l'exercice normal de leurs fonctions ou avec l'autorisation du/de la Secrétaire général(e), ils ne doivent communiquer à qui que ce soit, gouvernement, entité, personne ou toute autre source, aucun renseignement dont ils ont eu connaissance du fait de leur situation officielle et dont ils savent ou devraient savoir qu'il n'a pas été rendu public. La cessation de service ne les dégage pas de ces obligations». 
d'identification des personnes porteraient atteinte au droit d'accès aux procédures d'asile. De plus, des mesures de sécurité nationale qui imposent un partage des données sur les réfugiés iraient à l'encontre de leur droit à la vie privée et de leur droit à la sécurité. Le suivi de ces évolutions, l'analyse de leur impact sur les réfugiés et les demandeurs d'asile sont devenus des défis importants pour le HCR. Sans oublier qu'il faut s'assurer que le plaidoyer soit fait en leur nom et que de telles mesures ne compromettent pas indûment le respect des droits des réfugiés et des demandeurs d'asile. Ceci justifie le besoin d'établir au sein du HCR une expertise sur les technologies de traitement des données et sur la protection de la vie privée. Devant l'évolution rapide de ce phénomène et la multiplication des interrogations qui y sont liées, ainsi que l'ampleur des défis auxquels le HCR doit faire face, il semble indispensable que ce dernier se tourne de plus en plus vers des experts de la protection des données et de la vie privée. 